\title{
EGRET Observations of the Diffuse Gamma-Ray Emission in Orion: Analysis Through Cycle 6
}

\author{
S. W. Digel, ${ }^{1}$ E. Aprile, ${ }^{2}$ S. D. Hunter,${ }^{3}$ R. Mukherjee, ${ }^{4}$ and F. Xu ${ }^{2}$ \\ 1. USRA, NASA/Goddard Space Flight Center, Code 660.2, Greenbelt, MD 20771 \\ 2. Physics Dept. \& Columbia Astrophysics Lab., Columbia University, New York, NY 10027 \\ 3. NASA/Goddard Space Flight Center, Code 661, Greenbelt, MD 20771 \\ 4. Dept. of Physics \& Astronomy, Barnard College, Columbia University, New York, NY 10027
}

\begin{abstract}
We present a study of the high-energy diffuse emission observed toward Orion by the Energetic Gamma-Ray Experiment Telescope (EGRET) on the Compton Gamma-Ray Observatory. The total exposure by EGRET in this region has increased by more than a factor of two since a previous study. A simple model for the diffuse emission adequately fits the data; no significant point sources are detected in the region studied $\left(l=195^{\circ}\right.$ to $220^{\circ}$ and $b=-25^{\circ}$ to $\left.-10^{\circ}\right)$ in either the composite dataset or in two separate groups of EGRET viewing periods considered. The gamma-ray emissivity in Orion is found to be $(1.65 \pm 0.11) \times 10^{-26} \mathrm{~s}^{-1} \mathrm{sr}^{-1}$ for $E>100 \mathrm{MeV}$, and the differential emissivity is well-described as a combination of contributions from cosmic-ray electrons and protons with approximately the local density. The molecular mass calibrating ratio is $N\left(\mathrm{H}_{2}\right) / W_{\mathrm{CO}}=(1.35 \pm 0.15) \times 10^{20} \mathrm{~cm}^{-2}\left(\mathrm{~K} \mathrm{~km} \mathrm{~s}^{-1}\right)^{-1}$.
\end{abstract}

Subject headings: gamma rays: observations — ISM: molecules — radio lines: ISM

\section{Introduction}

This paper presents an analysis of the most extensive data set to date of the Orion region obtained by the Energetic Gamma Ray Experiment Telescope (EGRET) on the Compton Gamma Ray Observatory (CGRO). Study of the diffuse high energy $(E>30 \mathrm{MeV})$ gamma-ray emission from nearby, massive interstellar clouds permits testing the mechanisms of gamma-ray production and measuring the local cosmic-ray (CR) density as well as properties of the interstellar medium (ISM). The goals of this work are to determine the high-energy CR density in Orion, the molecular mass calibrating factor $X \equiv N\left(\mathrm{H}_{2}\right) / W_{\mathrm{CO}}$, and to identify any point sources or resolved variations in $\mathrm{CR}$ density or $X$ within the Orion AB-Mon R2 complex of interstellar clouds. The Orion region was previously studied by Digel, Hunter, \& Mukherjee (1995; hereafter DHM) using EGRET data through 1993 of CGRO. Since the time of the earlier work by DHM, the overall exposure of EGRET toward the Orion region has increased by more than a factor of two, and has become 
much more uniform. As EGRET is now nearing the end of the life of its spark chamber gas, the currently-available observations represent essentially all the exposure that EGRET will obtain toward Orion.

Most of the motivations for studying the diffuse gamma-ray emission in Orion remain the same. The interstellar clouds in Orion, comprising the Orion A and B clouds and the more distant Mon R2 cloud, are the nearest giant molecular clouds ( $\sim 500 \mathrm{pc})$, with a mass $\sim 4 \times 10^{5} \mathrm{M}_{\odot}$ (Maddelena et al. 1986). The clouds are well-resolved by EGRET, and are far from the plane in the outer Galaxy, so their gamma-ray emission can be studied essentially in isolation from the general diffuse emission of the Milky Way.

Since the time of the previous work, more conservative cuts on zenith angle to reject earth albedo gamma rays were adopted for the standard EGRET data products. This decreases the number of photons, and the exposure, somewhat for each viewing period. We investigate here whether this change alters the findings of DHM.

To verify the production mechanisms of gamma rays in interstellar clouds, and to determine the CR densities for individual molecular clouds, independent measurements of the interstellar matter distribution are required. The atomic hydrogen phase of the ISM is observable via the characteristic $21 \mathrm{~cm}$ line radiation. However, molecular hydrogen $\mathrm{H}_{2}$ generally cannot be directly observed at interstellar conditions. The standard tracer of the large-scale distribution of $\mathrm{H}_{2}$ is the $J=1-0$ line of $\mathrm{CO}$ at $115 \mathrm{GHz}$. CO is the second most abundant interstellar molecule after $\mathrm{H}_{2}$, tends to form under the same conditions, and is excited to the $J=1$ rotational state by collisions with $\mathrm{H}_{2}$. The relation between $N\left(\mathrm{H}_{2}\right)$ and $W_{\mathrm{CO}}$, the integrated intensity of the CO line, is empirically known to be approximately a proportionality; the proportionality constant $N\left(\mathrm{H}_{2}\right) / W_{\mathrm{CO}}$ is denoted $X$. All determinations of $X$ require an indirect tracer of $N\left(\mathrm{H}_{2}\right)$. Helium and heavier elements are assumed to be distributed like the hydrogen, as is commonly done in studies of diffuse gamma-ray emission; the emissivities referred to in other sections of this paper are therefore the effective rates per hydrogen atom. Owing to the penetration of clouds by high-energy CRs, and the transparency of the ISM to gamma rays, gamma-ray intensity can be used as such a tracer. Here we use the gamma-ray emission from Orion to calibrate the $X$-ratio and thereby infer the CR densities in the Orion neighbourhood.

The recent reports of an extended region of carbon and oxygen nuclear line emission in Orion from the COMPTEL instrument on CGRO (Bloemen et al. 1997) are another motivation for an updated study of the gamma-ray observations by EGRET. To explain the observed flux of the ${ }^{12} \mathrm{C}^{*}$ and ${ }^{16} \mathrm{O}^{*}$ lines at $4.44 \mathrm{MeV}$ and $6.13 \mathrm{MeV}$, respectively, a large enhancement of low-energy ( $<100 \mathrm{MeV} /$ Nucleon) CRs is needed. Note, however, that a recent re-evaluation of the COMPTEL background has shown that the Orion result was largely spurious (Bloemen et al. 1999). The CR enhancement factor depends on the amount of interstellar gas and hence on the $X$-ratio determined from EGRET analysis. 


\section{Data}

\section{1. $\quad \mathrm{H} \mathrm{I}$ and $\mathrm{CO}$}

We use the same $21 \mathrm{~cm} \mathrm{H} \mathrm{I}$ and $2.6 \mathrm{~mm}$ CO maps as DHM. Briefly, the H I surveys of Weaver \& Williams (1973) and Heiles \& Habing (1974) were combined and column densities $N(\mathrm{HI})$ were derived on the assumption of a uniform spin temperature of $125 \mathrm{~K}$. The CO surveys of Maddalena et al. (1986) and Huang et al. (1985), as combined in Dame et al. (1987), were used to derive the map of integrated intensity in the CO line, $W_{\mathrm{CO}}$. The region of interest for the present study is $l=195^{\circ}$ to $220^{\circ}$ and $b=-25^{\circ}$ to $-10^{\circ}$, although a $15^{\circ}$ wide border surrounding this area was also included in the $\mathrm{CO}$ and $\mathrm{H}$ I datasets to permit convolution with the broad PSFs (point spread functions) of EGRET in the central region. In directions where no CO data are available, principally $b<-25^{\circ}$, we assume $W_{\mathrm{CO}}=0$. For both $\mathrm{H}$ I and $\mathrm{CO}$ emissions, only one spectral line is evident along lines of sight in the region of interest; although the $21 \mathrm{~cm}$ line emission in particular has broad tails in velocity, all of the interstellar gas along the line of sight is assumed to be associated with Orion (i.e., have the same density of CRs) in the analysis below. The $N(\mathrm{HI})$ and $W_{\mathrm{CO}}$ maps were produced on the same grid used for binning the gamma-ray photons.

\subsection{Gamma-Ray}

We combine the data (photon counts and exposure maps) from all EGRET viewing periods with exposure within the region of interest $\left(l=195^{\circ}\right.$ to $220^{\circ}, b=-25^{\circ}$ to $-10^{\circ}$; Table 1$)$ and a $15^{\circ}$ border around this region. Only the area within $30^{\circ}$ of the pointing direction for any given viewing period was included in the combined datasets. The sensitivity of EGRET for inclination angles greater than $30^{\circ}$ is relatively poor, so few photons and little exposure are lost. The advantage of making this truncation is that the relatively broad PSF far off axis (Thompson et al. 1993) need not be considered in the analysis; for each energy range only a single effective PSF is needed for the entire dataset. The gamma-ray data are binned on a $30^{\prime}$ grid in Galactic coordinates for this analysis. Details of the instrument design are discussed in Hughes et al. (1980) andKanbach et al. (1988), and the preflight and the postflight calibrations are described by Thompson et al. (1993) and Esposito et al. (1999).

In the analysis described below, the EGRET data are analyzed for six broad energy ranges spanning 30-10,000 MeV, and two integral ranges (energy $E>100 \mathrm{MeV}$ and $E>300 \mathrm{MeV}$ ). For each range, the corresponding exposure maps were derived on the assumption of an $E^{-2.1}$ input spectrum. The intensity maps (photon counts divided by exposure) for viewing periods with large overlaps and good exposure in the region of interest were intercompared to check their relative intensity calibrations. The seven viewing periods (1.0, 2.1, 337.0, 413.0, 419.5, 420.0, and 616.1) were compared on just four broad energy ranges (30-100, 100-300, 300-1000, and 1000-10,000 MeV) to improve the statistics of the comparisons. The relative calibrations were in good agreement 
except for viewing periods 2.1 and 616.1, which were found to be significantly brighter and fainter than the average, respectively. The correction factors were largest for viewing period (VP 616.1), ranging up to 1.9 on $30-100 \mathrm{MeV}$. For this late VP, the performance of the spark chamber had degraded significantly; EGRET was operated in the narrow field of view mode during this VP, and so the overall effect on the composite data set is small.

Table 1 lists the numbers of photons and mean exposure (scaled as described above) for the representative energy ranges $E>100 \mathrm{MeV}$ and $E>300 \mathrm{MeV}$. The correction factors described above have been incorporated into Table 1 . The viewing periods listed in the table are grouped by observation date to show the two subsets that were considered below to check consistency with the analysis of DHM and to search for flaring point-source emission that might have been more significant in the viewing periods obtained since that work. The total number of photons for $E>100 \mathrm{MeV}$ in the region of interest is 10,257, compared to 5266 photons in DHM. (With the more restrictive cuts used here for Earth albedo gamma rays, the previous total for the same viewing periods is 4879.) The overall mean exposure has increased from $5.9 \times 10^{8} \mathrm{~cm}^{2} \mathrm{~s}$ (before the more restrictive zenith angle cuts were adopted) to $13.5 \times 10^{8} \mathrm{~cm}^{2} \mathrm{~s}$.

\section{Analysis}

We use the same model as DHM for the emission in Orion, one that has been applied in several studies of diffuse gamma rays dating from the work of Lebrun et al. (1982). Under the assumption that high-energy CR electrons and protons uniformly penetrate the atomic and molecular gas in Orion, with the same densities in both phases, the distribution of photon counts may be modeled as a linear combination of the $N(\mathrm{HI})$ and $W_{\mathrm{CO}}$ maps. In principle, allowance must also be made for inverse-Compton emission and gamma-ray production on ionized gas. If the CR density were uniform, the distribution of gamma-ray photons observed for some energy range could be written as

$$
\Theta(l, b)=A N(\mathrm{H} \mathrm{I})_{c}+B W(\mathrm{CO})_{c}+C N(\mathrm{H} \mathrm{II})_{c}+I C_{c}+\Sigma\left(D_{i} \delta_{l_{i} b_{i}}\right)+F \epsilon_{c}
$$

Here we have taken the finite spatial resolution of EGRET into account by convolving the predicted distribution of gamma-ray photons with the effective PSF of EGRET for the corresponding energy range. The subscript $c$ indicates multiplication by the exposure map and convolution with the effective PSF, as explained in DHM. $\epsilon_{c}$ is the exposure map itself convolved with the effective PSF for that energy range. In Eqn. (1), the coefficient $A$ is the emissivity (photons s $\mathrm{s}^{-1} \mathrm{sr}^{-1}$ ) per hydrogen atom, $B=2 A X$, where $X=N\left(\mathrm{H}_{2}\right) / W_{\mathrm{CO}}, C$ is the emissivity of the ionized hydrogen, $D_{i}$ are the numbers of photons from each point source for that energy range, and $F$ is the isotropic diffuse emission. For the Orion region, the IC emission and contributions from CR interactions with ionized hydrogen are expected to contribute at only the several percent level, and largely in a smooth way that can be subsumed with the isotropic emission (DHM). Under these assumptions 
and approximations Eqn. (1) may be re-written as

$$
\Theta(l, b)=A N(\mathrm{H} \mathrm{I})_{c}+B W(\mathrm{CO})_{c}+C \epsilon_{c}+\Sigma\left(D_{i} \delta_{l_{i} b_{i}}\right)
$$

We use the maximum likelihood method (Mattox et al. 1996) to fit the model (Eqn. 2) to the binned photon data for the various energy ranges and combinations of viewing periods considered. The likelihood value, $L$, for a given set of parameter values is the product of the probabilities that the measured photon counts are consistent with the predicted counts in each pixel on the assumption of Poisson statistics. The probability of one model with likelihood $L_{1}$ better representing the data than another model with likelihood $L_{2}$ is determined from twice the difference of the logarithms of the likelihoods, $2\left(\ln L_{2}-\ln L_{1}\right)$. This difference, referred to as the test statistic $T S$, is distributed like $\chi^{2}$ in the null hypothesis, with the number of degrees of freedom being the difference between the number of free parameters in the two models. To fit the model to the observations, the positions and fluxes of the point sources and the values of the other coefficients in Eqn. (2) are adjusted to maximize the likelihood. The strength of the dependence of the likelihood function on the various parameters of the model permits their uncertainties or significances to be estimated quantitatively. Values for the different coefficients in Eqn. (1) can be determined separately as long as their corresponding maps are distinguishable, i.e., are linearly independent. Figure 1 shows that the maps of $N(\mathrm{HI})_{c}, W(\mathrm{CO})_{c}$, and $\epsilon_{c}$ for the Orion region are distinguishable, for the representative energy range $E>100 \mathrm{MeV}$. The EGRET exposure to the region modeled is not uniform, having a gradient with longitude, so the sensitivity decreases at higher longitudes and lower latitudes. The exposure variations are accounted for in the maximum likelihood analysis, in the sense that regions with greater exposure effectively have greater weight.

We first used the maximum likelihood method to systematically search for gamma-ray point sources in the region of interest. The point source search entails determining the maximum likelihood values of the parameters in Eqn. (2) for an assumed point source at each position in the $30^{\prime}$ grid, generating a map of $T S$. Owing to the strong energy dependence of the effective PSF of EGRET, in application this test is most sensitive if TS maps for subranges of energy are generated separately, then added together (Mattox et al. 1996). Mattox et al. (1996) show that

for the case of six combined maps, the values of $T S$ for the point source search are distributed as $\chi^{2}$ with 8 degrees of freedom in the null hypothesis.

\section{Results}

A maximum likelihood search for point sources was made for the two groups of viewing periods identified in Table 1, as well as for the sum of all viewing periods. No significant source detections, greater than 4- $\sigma$ statistical significance, were found in any of the groups of viewing periods or for any of the energy ranges analyzed. Figure 2 shows the sum of the TS maps for the 30-100, 100-150, 150-300, 300-500, 500-1000, and 1000-10,000 MeV ranges for the combined set of 
all viewing periods. The peak value of 31.2 , near $\left(195.0^{\circ},-19.5^{\circ}\right)$, corresponds to a significance of $3.8 \sigma$.

The extended feature associated with this peak (Fig. 2) largely originates in the $30-100 \mathrm{MeV}$ TS map. In this low energy range, the effective PSF of EGRET is quite broad and the feature could represent the presence of a soft source or sources just outside the region of interest. In fact, the Third EGRET catalog (3EG) (Hartman et al. 1999) includes two point sources just below the lower longitude limit: 3EG J0459+0544 $\left(193.99^{\circ},-21.66^{\circ}\right)$ and 3EG J0530+1323 $\left(191.50^{\circ}\right.$, $\left.-11.09^{\circ}\right)$.

The extended excess near $\left(215^{\circ},-19^{\circ}\right)$ in Figure 2 has a peak TS value of 24.0, corresponding to a statistical significance of $3.0 \sigma$. However, the overall significance of the extended excess is less; the integrated residual intensity for $E>100 \mathrm{MeV}$ (Fig. 3c) corresponds to approximately only 25 photons in a region where 280 are expected. The 3EG catalog (Hartman et al. 1999) contains no sources consistent with this extended region, and a search of NED reveals no likely counterparts at other wavelengths. We note that position of this excess is consistent with two sources in the Second EGRET catalog (Thompson et al. 1995), GRO J0545-1156 and GRO J0552-1028. However, both of these sources were below threshold in the 3EG catalog analysis.

In their earlier analysis of the same region that we model here, DHM reported the detection of three marginal sources at a statistical significance of $\sim 3 \sigma$. Two of these sources were probably the same as the unidentified sources GRO 0605-09 $\left(l=216.6^{\circ}, b=-14.4^{\circ}\right)$ and GRO 0546-02 $\left(l=207.6^{\circ}, b=-15.6^{\circ}\right)$ listed in the First EGRET catalog (Fichtel et al. 1994). The third source at $l=203.0^{\circ}, b=-17.5^{\circ}$ had not been detected by EGRET previously. None of the above three sources were detected in the current analysis of the complete data set.

Since we are primarily interested in the diffuse gamma-ray emission here, we investigated how the maximum likelihood values of the parameters $A, B$, and $C$ that describe the diffuse emission (Eqn. 2) depended on the number and positions of point sources in the model. The cases investigated included the following: no point sources, a hypothetical source at $\left(215^{\circ},-19^{\circ}\right)$, and the two 3EG sources mentioned above. For all of these cases, the maximum likelihood values of the diffuse parameters were the same within 1- $\sigma$, except in the 30-100 $\mathrm{MeV}$ range, where the inclusion of the two 3EG sources improved the fit and changed the best-fitting $B$ and $C$ terms substantially. Although these sources were not detected with strong significance, which is not unexpected as they are outside the region we model, we included them in the model that we adopted for all of the analysis described below.

Figure 3 shows the EGRET observations, the maximum likelihood best-fitting model, and the residual map for the $E>100 \mathrm{MeV}$ energy range using all of the EGRET data for Orion. The gamma-ray intensities were obtained by dividing the photon map used to fit the parameters by the exposure map. The good agreement between the observed intensities and the model for $E>100$ $\mathrm{MeV}$, calculated using the parameters for the combined groups in Table 2, is demonstrated in the residual map shown in Figure $3 c$. The figure shows that the model intensity map clearly has no 
large-scale deviation from the observed intensity. Owing to the inclusion of the two low-longitude sources, the residual intensities near longitude $195^{\circ}$ are small. The remaining extended residual was discussed above.

Figure 3 also shows that there is no significant deviation from the fit in the region of Mon R2. This indicates that although Mon R2 is 300 pc more distant than Orion (Maddalena et al. 1986), its properties can be considered to be the same as those of the clouds in Orion. Further, we note that the residual intensity shown in Figure $3 c$ is not correlated with $W_{\mathrm{CO}}, N(\mathrm{H} \mathrm{I})$, or the total column density of interstellar gas. The absence of the correlation with $W_{\mathrm{CO}}$ is consistent with the $X$-ratio being independent of $W_{\mathrm{CO}}$ and of the position in Orion. There is no statistically significant variation of the $X$-ratio and emissivity between Orion $\mathrm{A}$, Orion $\mathrm{B}$, and Mon $\mathrm{R} 2$ molecular clouds. The lack of correlation between the residual intensity and the interstellar gas is consistent with the assumption that the atomic and molecular gas is uniformly permeated by CRs, and that the CR density is uniform. The model (Eqn. 2) therefore provides an adequate description of the high-energy gamma-ray emission from the Orion region.

The parameter values for the model fits to the combined EGRET data and their uncertainties are listed in Table 2. No significant differences are seen from the results of DHM. The uncertainties in the model parameter values have decreased as expected owing to the greatly improved exposure. Interpretations of the values of the parameters are discussed in the following paragraphs.

Figure $4 a$ shows the differential $\gamma$-ray emissivity derived from the coefficient $A$ of the model fit to each of the six energy ranges in Table 2 . As the figure illustrates, the general energy dependence of the emissivity is well described by the electron-Bremsstrahlung $(e-B)$ (Koch \& Motz 1959; Fichtel et al. 1991) and nucleon-nucleon $(n-n)$ (Stecker 1989) production functions parameterized by Bertsch et al. (1993) for the solar vicinity. The deviation from the Bertsch et al. production function in the 1000-10,000 MeV range has been seen in other studies of Galactic diffuse emission with EGRET data (e.g., Hunter et al. 1994; DHM; Digel et al. 1996; Hunter et al. 1997). This deviation is not seen in studies of the isotropic emission at high latitudes (e.g., Sreekumar et al. 1998) and therefore is unlikely to represent a high-energy calibration error. The most plausible interpretation, that the calculation of gamma-ray production from $n-n$ interactions somewhat underestimates the yield (Hunter et al. 1997), does not affect the results presented here.

The integral gamma-ray emissivity in Orion is found to be $(1.65 \pm 0.11) \times 10^{-26} \mathrm{~s}^{-1} \mathrm{sr}^{-1}$ for $E>100 \mathrm{MeV}$, confirming the value obtained in the earlier DHM analysis. It compares well with the values obtained for the Galactic plane in the solar vicinity in large-scale studies of diffuse emission (e.g., Lebrun \& Paul 1985; Strong et al. 1988; Strong \& Mattox 1996), which range from $(1.54-1.8) \times 10^{-26} \mathrm{~s}^{-1} \mathrm{sr}^{-1}$. However, studies of individual clouds within $\sim 500 \mathrm{pc}$ with EGRET data yield a wider range of integral emissivities: $(2.4 \pm 0.2) \times 10^{-26} \mathrm{~s}^{-1} \mathrm{sr}^{-1}$ in Ophiuchus (Hunter et al. 1994), $(2.01 \pm 0.15) \times 10^{-26} \mathrm{~s}^{-1} \mathrm{sr}^{-1}$ in the local clouds toward Monoceros (Digel et al. 1999), and $(1.84 \pm 0.10) \times 10^{-26} \mathrm{~s}^{-1} \mathrm{sr}^{-1}$ in the local clouds toward Cepheus (Digel et al. 1996). The range of emissivities, which we note decrease with increasing Galactocentric distance of the 
cloud, suggests a fairly steep gradient of CR density at the solar circle that is smoothed in the large-scale studies, which typically have resolutions of $2 \mathrm{kpc}$ or more.

The $X$-ratios in Table 2 are derived from the values of $A$ and $B$ for each energy range and are shown in Figure $4 b$. As expected for an intrinsic property of the molecular clouds, the value of $X$ does not vary significantly with energy, except possibly for a decrease in the 1000-10,000 MeV range. The reason for the marginally-significant decrease at the highest energies is not clear, as the highest-energy CRs should not be excluded from the dense, molecular parts of the clouds.

The value of $X$ derived for the $E>100 \mathrm{MeV}$ range, $(1.35 \pm 0.15) \times 10^{20} \mathrm{~cm}^{-2}[\mathrm{~K} \mathrm{~km}$ $\left.\mathrm{s}^{-1}\right]^{-1}$, is adopted here as the best overall value, in terms of the numbers of photons and the resolution of the gamma-ray observations, from our analysis. The non-uniformity of the exposure across the field (Fig. 1c) means that this should be considered an exposure-weighted average, or more properly an exposure-and-total column density weighted average. As mentioned in $\S 3$, the likelihood function is most sensitive to the model in regions with the most photons, where the exposure and gas column density are greatest. The exposure difference between the Orion A and B clouds is only about 20\% (Fig. 1c), however, and the residual map in Figure $3 c$ indicates that the same $X$-ratio applies to both clouds within the resolution and statistics of the data. The value of $X$ reported in DHM, $(1.06 \pm 0.14) \times 10^{20} \mathrm{~cm}^{-2}\left[\mathrm{~K} \mathrm{~km} \mathrm{~s}^{-1}\right]^{-1}$, is marginally less than the value found here. Owing to the greatly-improved uniformity of exposures in the dataset analyzed here, we consider the new finding to be the more reliable.

The emissivities and $X$-ratios we find for the Orion region are compared in Table 3 with results from earlier studies. The studies of Bloemen et al. (1984) and Houston \& Wolfendale (1985) were based on COS-B data, and the findings have been scaled here to the updated CO radiation temperature scale of Bronfman et al. (1988). DHM found a value of $X$ much lower than that reported by Bloemen et al. (1984), and the lower value is confirmed here. The instrumental background of COS-B was significant, and had structure on the same angular scale as the molecular clouds in Orion. The final background corrections were not available at the time of the analysis by Bloemen et al. (1984), and in any case small errors in the corrections for the different COS-B viewing periods would have had a large effect on the value of $X$ derived. The integral gamma-ray emissivities in Table 3 are approximately consistent across the various studies.

The differential spectrum of the isotropic intensity inferred from the coefficients $C$ in Table 2 is shown in Figure 5. The integrated intensity for $E>100 \mathrm{MeV}$ is $(1.46 \pm 0.23) \times 10^{-5} \mathrm{~cm}^{-2}$ $\mathrm{s}^{-1}$. The overall average spectrum of the isotropic emission found by Sreekumar et al. (1998), also shown in Figure 5, has an integral intensity of $(1.45 \pm 0.05) \times 10^{-5} \mathrm{~cm}^{-2} \mathrm{~s}^{-1}$ and a spectral index of $2.10 \pm 0.03$. On consideration of the statistical uncertainties, the intensity found here is consistent with the expected intensity of the isotropic emission together with the intensity of inverse Compton emission and gamma-ray production on ionized hydrogen, which were neglected in the model (see $\S 3$ ). 


\section{Conclusions}

This analysis of the EGRET data for the Orion region essentially confirms the findings of the earlier work by DHM based on much less data. No significant point sources are detected in any of four groups of viewing periods or in the combined dataset; the marginal sources reported by DHM are no longer even marginally significant. The emissivity and $X$-ratio derived for the diffuse emission are not significantly affected if a point source is included at the position of the greatest remaining residual intensity. A simple linear model for the gamma-ray emission, with adjustable parameters for the gamma-ray emissivity, the $X$-ratio, and the isotropic intensity, including two 3EG sources just outside the field, is found to fit the observations adequately across the EGRET energy range. The gamma-ray emissivity in Orion is consistent with that found for the solar circle in large-scale studies of diffuse emission, and its value relative to emissivities for other clouds in the solar vicinity suggests a fairly strong gradient of CR density with Galactocentric distance at the solar circle. The spectrum of emissivity is consistent with electron and proton CR spectra approximately the same as in the solar vicinity. The molecular mass-calibrating $X$-ratio is $(1.35 \pm 0.15) \times 10^{20} \mathrm{~cm}^{-2}\left(\mathrm{~K} \mathrm{~km} \mathrm{~s}^{-1}\right)^{-1}$, and the gamma-ray emissivity for $E>100 \mathrm{MeV}$ is $(1.65 \pm 0.11) \times 10^{-26} \mathrm{~s}^{-1} \mathrm{sr}^{-1}$.

This research has made use of the NASA/IPAC Extragalactic Database (NED) which is operated by the Jet Propulsion Laboratory, under contract with the National Aeronautics and Space Administration. The authors wish to thank Hans Bloemen for his comments on the manuscript. SWD acknowledges support from the CGRO Guest Investigator Program grant NAG5-2823. EA acknowledges support from the CGRO Guest Investigator Program grant NAG5-2872. RM acknowledges support from the CGRO Guest Investigator Program grant NAG5-3696. 


\section{REFERENCES}

Bertsch, D. L., et al. 1993, ApJ, 416, 587

Bloemen, H., et al. 1999, in preparation.

Bloemen, H., et al. 1997, ApJ, 475, L25

Bloemen, J. B. G. M., et al. 1984, A\&A, 139, 37

Bronfman, L., Cohen, R. S., Alvarez, H., May, J., \& Thaddeus, P. 1988, ApJ, 324, 248

Dame, T. M., et al. 1987, ApJ, 322, 706

Digel, S. W., Hunter, S. D., and Mukherjee, R. 1995, ApJ, 441, 270

Digel, S. W., et al. 1996, ApJ, 463, 609

Digel, S. W., et al. 1999, in prep.

Esposito, J., et al. 1999, ApJ, submitted.

Fichtel, C. E., et al. 1991, ApJ, 374, 134

Fichtel, C. E., et al. 1994, ApJS, 94, 551

Hartman, R. C., et al. 1999, ApJ, in press.

Heiles, C., \& Habing, H. J. 1974, A\&AS, 14, 1

Houston, B. P., \& Wolfendale, A. W. 1985, J. Phys. G, 11, 407

Huang, Y.-L. 1985, Ph. D. thesis, Columbia University

Hughes, E. B., et al. 1980, IEEE Trans. Nucl. Sci., NS-27, 364

Hunter, S. D., Digel, S. W., de Geus, E. J., \& Kanbach G. 1994, ApJ, 436, 216

Hunter, S. D., et al. 1997, ApJ, 481, 205

Kanbach, G., et al. 1988, Space Science Reviews, 49, 69

Koch, H. W., \& Motz, J. W. 1959, Rev. Mod. Phys., 31, 920

Lebrun, F., et al. 1982, A\&A, 107, 390

Lebrun, F., \& Paul, J. 1985, in Proc. 19th Internat. Cosmic-Ray Conf. (La Jolla)(NASA CP-2376), 1, 197

Maddalena, R. J., Morris, M., Moscowitz, J., \& Thaddeus, P. 1986, ApJ, 303, 375

Mattox, J. R., et al. 1996, ApJ, 461, 396

Sreekumar, P., et al. 1998, ApJ, 494, 523

Stecker, F. W. 1989, Cosmic Gamma Rays, Neutrinos and Related Astrophysics, ed. M. M. Shapiro \& J. P. Wefel (Dordrecht: Kluwer), 85

Strong, A. W., et al. 1988, A\&A, 207, 1

Strong, A. W., \& Mattox, J. R. 1996, A\&A, 308, L21 
Thompson, D. J., \& Fichtel, C. E. 1982, A\&A, 109, 352

Thompson, D. J., et al. 1993, ApJS, 86, 629

Thompson, D. J., et al. 1995, ApJS, 101, 259

Weaver, H., \& Williams, D. R. W. 1973, A\&AS, 8, 1 


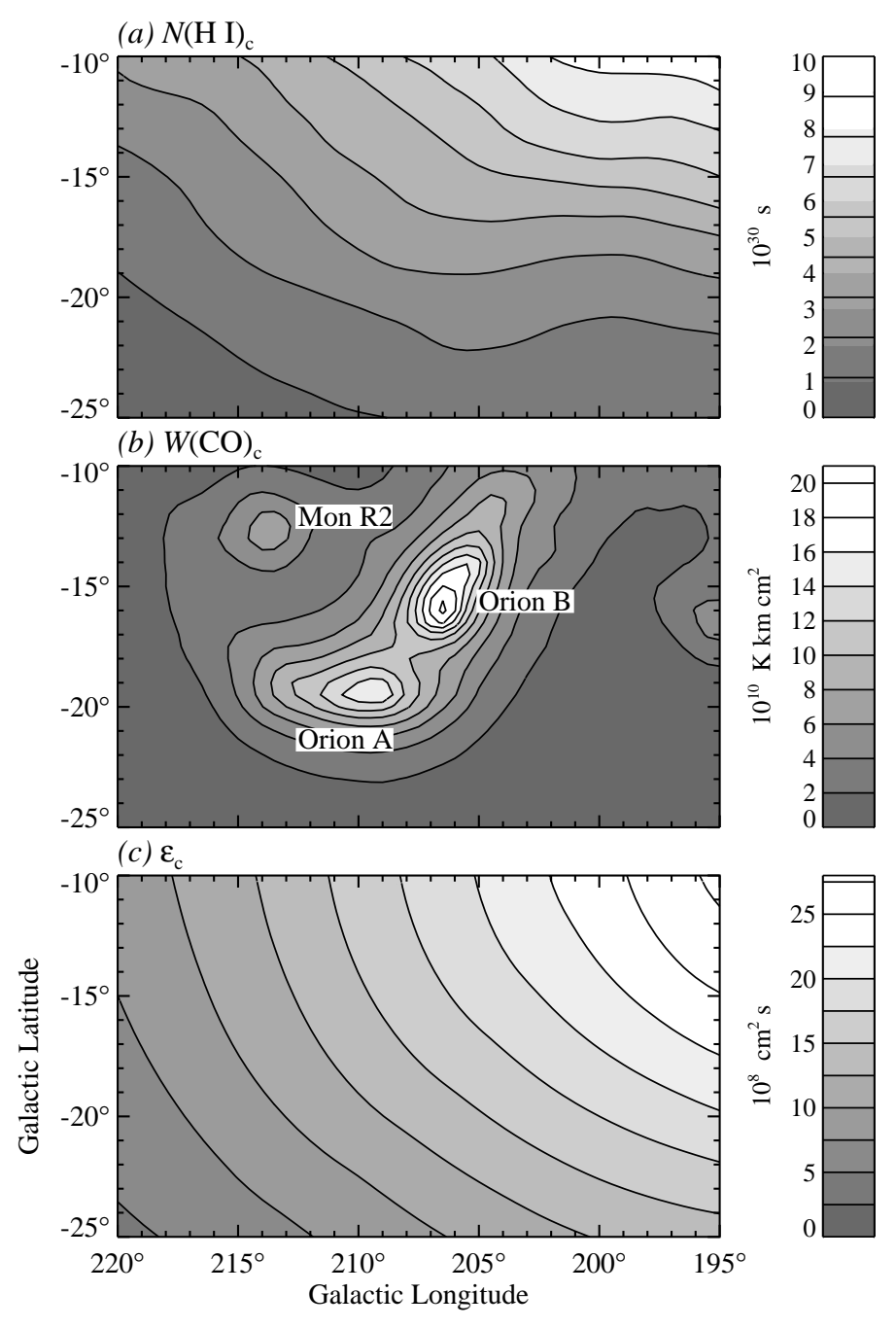

Fig. 1. - The maps $(a) N(\mathrm{HI})_{c}$, $(b) W(\mathrm{CO})_{c}$, and $(c) \epsilon_{c}$ described in the text, calculated for the energy range $E>100 \mathrm{MeV}$. 

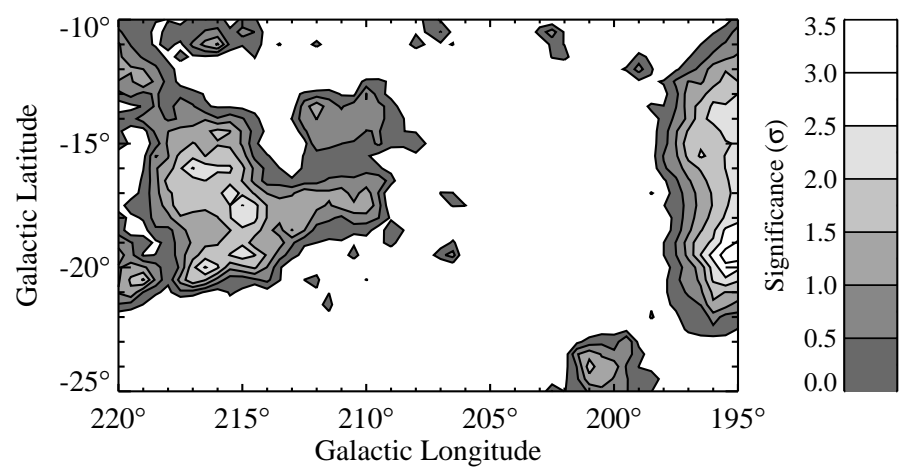

Fig. 2.- Composite map of likelihood test statistic $T S$, the sum of $T S$ maps for six energy ranges spanning $E=30-10,000 \mathrm{MeV}$ and representing the significance of a point source in the model at each position in the $30^{\prime}$ grid. The contours are in units of statistical significance in equivalent sigma, from 0.5 to 3.5 in steps of 0.5 . 
(a) Intensity

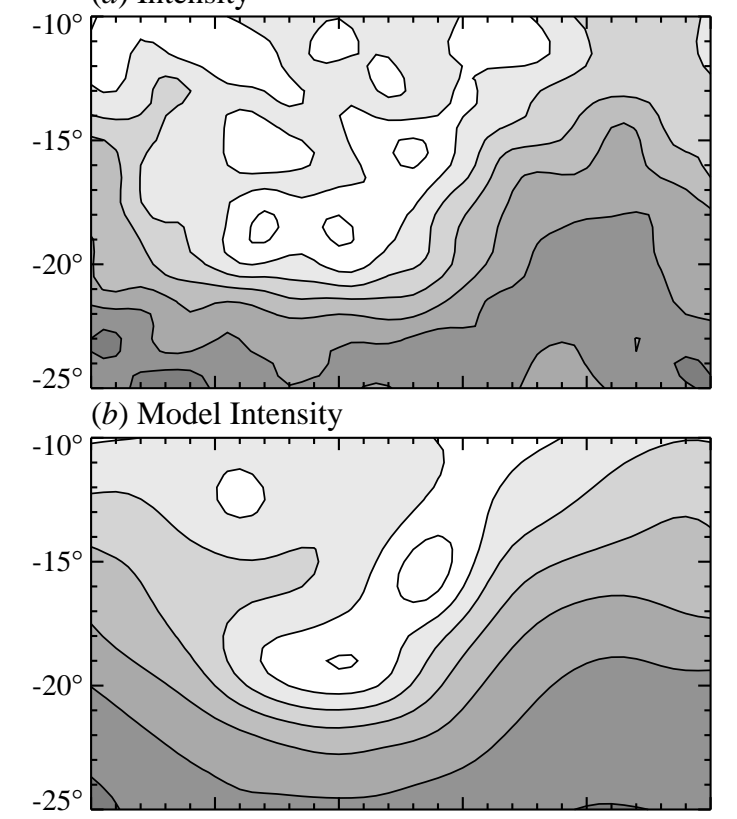

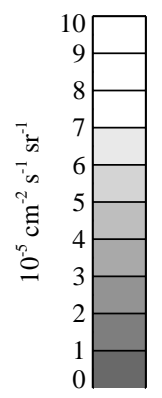

(c) Residual Intensity (Obs - Model)

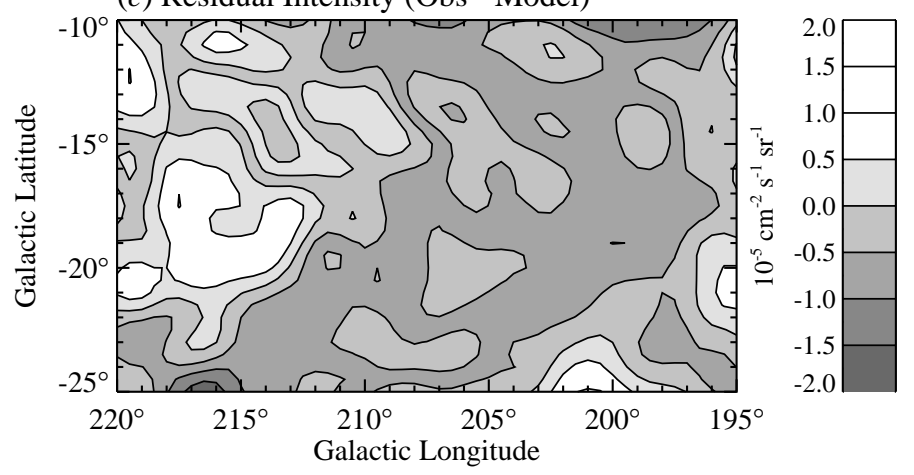

Fig. 3.- (a) Observed gamma-ray intensity (all viewing periods in Table 1), (b) maximum likelihood model (Eqn. 2 with the coefficients from Table 2), and (c) residual map (observed - model) for the energy range $E>100 \mathrm{MeV}$. The maps have all been smoothed slightly, by convolution with a 2-dimensional gaussian of FWHM $1.5^{\circ}$, to reduce the statistical fluctuations in the EGRET map. 

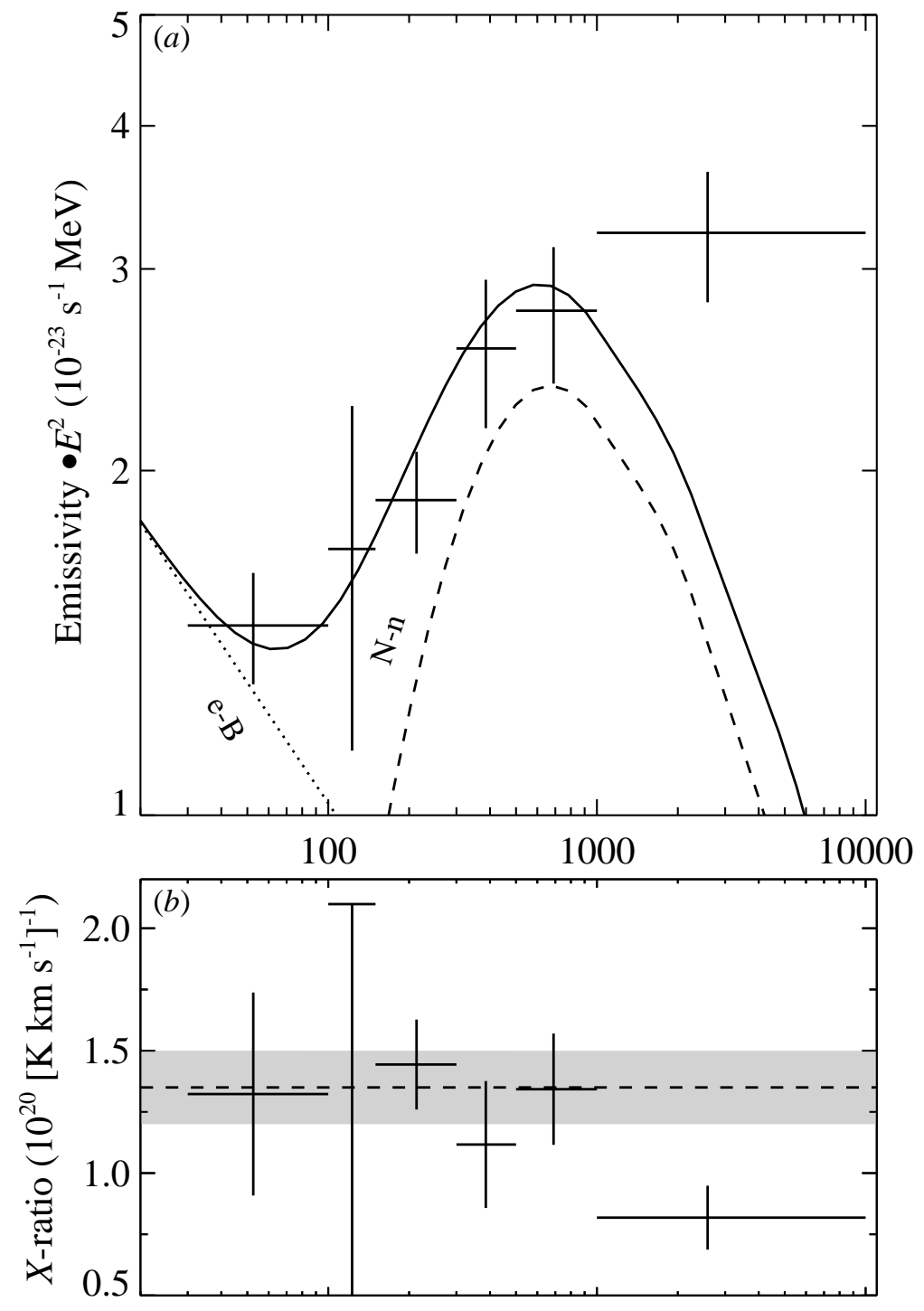

Fig. 4.- (a) Gamma-ray emissivity in the Orion region, derived from the coefficients $A$ in Table 2. The horizontal bars indicate the energy ranges and the vertical bars the $1 \sigma$ uncertainties of the parameters. The solid curve indicates the best-fitting linear combination of the electronBremsstrahlung $(e-B)$ and nucleon-nucleon $(n-n)$ production functions used by Bertsch et al. (1993), which are also shown separately. (b) The energy dependence of $X=B / 2 A$ (Table 2). For the $30-100 \mathrm{MeV}$ energy range, the $2-\sigma$ upper limit is shown. The dashed line and the gray shaded region indicate the $X$-ratio derived for the $E>100 \mathrm{MeV}$ range and its $1-\sigma$ uncertainty. 


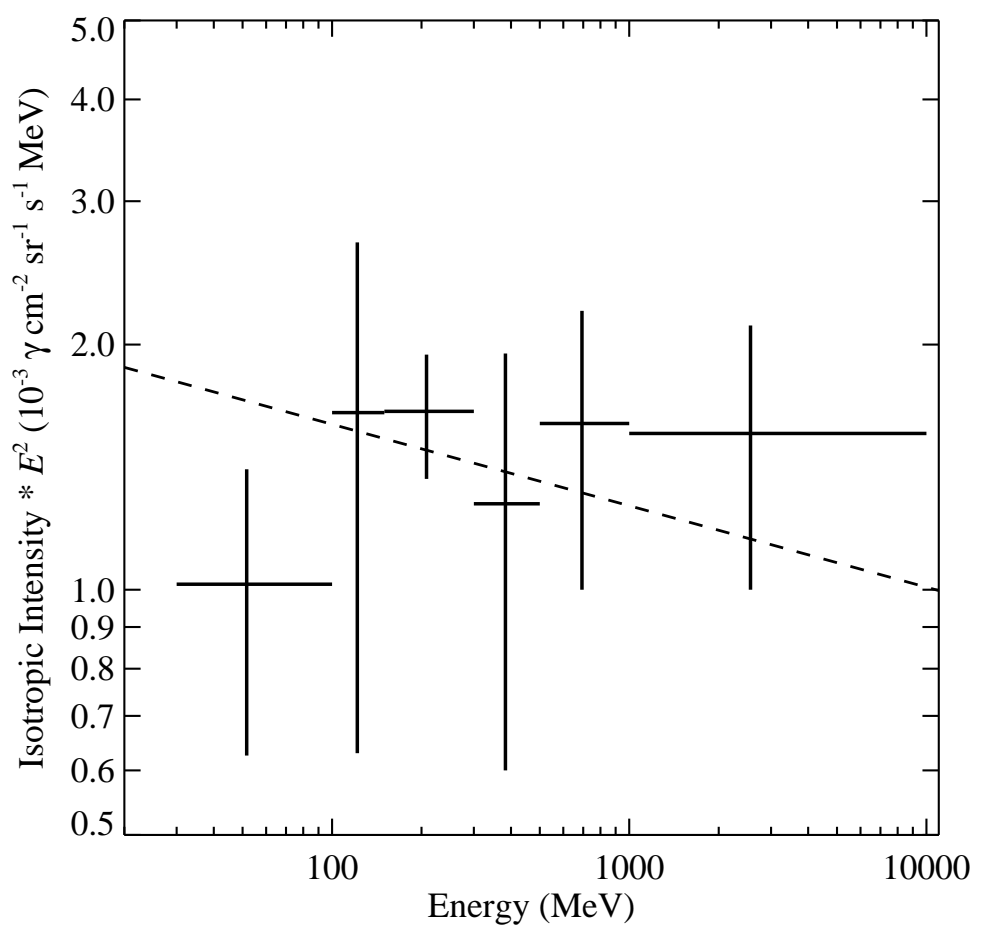

Fig. 5.- The spectrum of the isotropic intensity toward Orion, derived from the coefficients $C$ in Table 2. The dashed line is the overall average isotropic intensity derived by Sreekumar et al. (1998) for the high-latitude sky. See the text for the parameters of the spectra. 
TABLE 1

EGRET OBSERVATIONS TOWARD ORION

\begin{tabular}{|c|c|c|c|c|c|c|c|}
\hline \multirow{3}{*}{$\begin{array}{l}\text { VIEWING } \\
\text { PERIOD }\end{array}$} & \multirow[b]{3}{*}{ DATES } & \multirow{2}{*}{\multicolumn{2}{|c|}{ POINTING CENTER }} & \multicolumn{2}{|c|}{$E>100 M e V$} & \multicolumn{2}{|c|}{$E>300 M e V$} \\
\hline & & & & & \multirow{2}{*}{$\begin{array}{l}\text { Mean Exposure } \\
\qquad\left(10^{7} \mathrm{~cm}^{2} \mathrm{~s}\right)\end{array}$} & \multirow[b]{2}{*}{ Photons } & \multirow{2}{*}{$\begin{array}{l}\text { Mean Exposure } \\
\quad\left(10^{7} \mathrm{~cm}^{2} \mathrm{~s}\right)\end{array}$} \\
\hline & & $l$ & $b$ & Photons & & & \\
\hline $0.2 \ldots \ldots$ & 1991 Apr 22-Apr 28 & $186 .^{\circ} 0$ & $-3 .^{\circ} 3$ & 364 & 4.9 & 131 & 4.8 \\
\hline $0.3 \ldots \ldots$ & 1991 Apr 28-May 01 & 193.4 & -4.2 & 380 & 4.9 & 134 & 4.8 \\
\hline $0.4 \ldots \ldots$. & 1991 May 01-May 04 & 193.4 & -4.3 & 406 & 4.7 & 152 & 4.7 \\
\hline $0.5 \ldots \ldots$ & 1991 May 04-May 07 & 184.5 & -5.9 & 252 & 3.0 & 89 & 3.0 \\
\hline $1.0 \ldots \ldots$ & 1991 May 16-May 30 & 190.9 & -4.7 & 1407 & 17.7 & 519 & 17.4 \\
\hline $2.1 \ldots \ldots$. & 1991 Jun 08-Jun 15 & 194.9 & -7.3 & 942 & 10.2 & 386 & 10.1 \\
\hline $36.0 \ldots \ldots$. & 1992 Aug 11-Aug 12 & 169.8 & -11.4 & 4 & 0.1 & 2 & 0.1 \\
\hline $36.5 \ldots \ldots$. & 1992 Aug 12-Aug 20 & 168.2 & -9.5 & 16 & 0.2 & 9 & 0.2 \\
\hline $39.0 \ldots \ldots$. & 1992 Sep 01-Sep 17 & 167.2 & -9.2 & 14 & 0.2 & 5 & 0.2 \\
\hline $41.0 \ldots \ldots$. & 1992 Oct $08-$ Oct 15 & 228.0 & 2.8 & 151 & 1.7 & 58 & 1.7 \\
\hline $44.0 \ldots \ldots$. & 1992 Nov 03-Nov 17 & 228.0 & 2.8 & 276 & 3.1 & 113 & 3.2 \\
\hline $213.0 \ldots \ldots$. & 1993 Mar 23-Mar 29 & 182.6 & -8.2 & 130 & 1.6 & 40 & 1.6 \\
\hline $221.0 \ldots \ldots$ & 1993 May $13-$ May 24 & 187.5 & -5.9 & 311 & 3.5 & 135 & 3.5 \\
\hline $310.0 \ldots \ldots$ & 1993 Dec 01-Dec 13 & 195.1 & 4.3 & 302 & 4.1 & 137 & 4.3 \\
\hline $321.1 \ldots \ldots$ & 1994 Feb 08-Feb 15 & 181.4 & -2.6 & 187 & 2.3 & 78 & 2.2 \\
\hline $321.5 \ldots \ldots$. & 1994 Feb 15-Feb 17 & 181.4 & -2.6 & 49 & 0.8 & 22 & 0.7 \\
\hline $337.0 \ldots \ldots$. & 1994 Aug 09-Aug 29 & 205.0 & -13.0 & 1845 & 21.8 & 932 & 25.1 \\
\hline $412.0 \ldots \ldots$. & 1995 Feb 28-Mar 07 & 185.3 & 0.7 & 107 & 1.4 & 42 & 1.7 \\
\hline $419.1 \ldots \ldots$. & 1995 Apr 04-Apr 11 & 207.3 & -19.0 & 540 & 8.4 & 273 & 10.8 \\
\hline $419.5 \ldots \ldots$ & 1995 May 09-May 23 & 211.9 & -17.6 & 930 & 13.1 & 483 & 17.1 \\
\hline $420.0 \ldots \ldots$ & 1995 May 23-Jun 06 & 198.2 & -18.3 & 868 & 13.3 & 452 & 17.5 \\
\hline $426.0 \ldots \ldots$ & 1995 Aug 08-Aug 22 & 184.5 & -5.9 & 168 & 2.5 & 74 & 3.5 \\
\hline $502.0 \ldots \ldots$. & 1995 Oct $17-$ Oct 31 & 190.7 & -11.5 & 274 & 3.7 & 133 & 4.6 \\
\hline $526.0 \ldots \ldots$. & 1996 Jul 30-Aug 13 & 187.7 & -3.6 & 32 & 0.5 & 10 & 0.6 \\
\hline $527.0 \ldots \ldots$. & 1996 Aug 13-Aug 20 & 190.1 & -1.8 & 26 & 0.3 & 6 & 0.4 \\
\hline $616.1 \ldots \ldots$. & 1997 Feb 18-Mar 18 & 191.4 & -11.0 & 275 & 6.91 & 38 & 8.6 \\
\hline Totals ..... & & & & 10257 & 134.7 & 4553 & 152.4 \\
\hline
\end{tabular}


TABLE 2

MODEL PARAMETERS BY ENERGY RANGE

\begin{tabular}{|c|c|c|c|c|c|c|}
\hline $\begin{array}{l}\text { Energy Range } \\
(\mathrm{MeV})\end{array}$ & $\begin{array}{c}A \\
\left(10^{-26} \mathrm{~s}^{-1} \mathrm{sr}^{-1}\right)\end{array}$ & $\begin{array}{c}B \\
\left(10^{-6} \mathrm{~cm}^{-2} \mathrm{~s}^{-1} \mathrm{sr}^{-1}\right. \\
\left.\left[\mathrm{K} \mathrm{km} \mathrm{s}^{-1}\right]^{-1}\right)\end{array}$ & $\begin{array}{c}C \\
\left(10^{-6} \mathrm{~cm}^{-2} \mathrm{~s}^{-1} \mathrm{sr}^{-1}\right)\end{array}$ & $\begin{array}{c}F_{1}^{a} \\
\left(10^{-7} \mathrm{~cm}^{-2} \mathrm{~s}^{-1}\right)\end{array}$ & $\begin{array}{c}F_{2}^{a} \\
\left(10^{-7} \mathrm{~cm}^{-2}\right) \mathrm{s}^{-1}\end{array}$ & $\begin{array}{c}X^{b} \\
\left(10^{20} \mathrm{~cm}^{-2}\right. \\
\left.\left[\mathrm{K} \mathrm{km} \mathrm{s}^{-1}\right]^{-1}\right)\end{array}$ \\
\hline $100-10,000 \ldots \ldots$ & $1.65 \pm 0.11$ & $4.44 \pm 0.41$ & $14.6 \pm 2.3$ & $3.0 \pm 2.2$ & $<32$ & $1.35 \pm 0.15$ \\
\hline $300-10,000 \ldots \ldots$ & $0.71 \pm 0.10$ & $1.64 \pm 0.30$ & $4.46 \pm 1.88$ & $<2.3$ & $<11$ & $1.18 \pm 0.27$ \\
\hline $30-100 \ldots \ldots \ldots$. & $2.69 \pm 0.30$ & $7.03 \pm 2.23$ & $23.7 \pm 9.1$ & $39 \pm 15$ & $85 \pm 29$ & $1.32 \pm 0.44$ \\
\hline $100-150 \ldots \ldots \ldots$ & $0.45 \pm 0.15$ & $<1.7$ & $5.5 \pm 3.4$ & $<7.4$ & $<29$ & $\ldots^{c}$ \\
\hline $150-300 \ldots \ldots \ldots$ & $0.49 \pm 0.05$ & $1.40 \pm 0.18$ & $5.52 \pm 0.96$ & $<2.8$ & $<14$ & $1.44 \pm 0.23$ \\
\hline $300-500 \ldots \ldots \ldots$ & $0.27 \pm 0.04$ & $0.59 \pm 0.14$ & $1.7 \pm 0.9$ & $<1.3$ & $<6$ & $1.12 \pm 0.31$ \\
\hline $500-1000 \ldots \ldots .$. & $0.22 \pm 0.03$ & $0.58 \pm 0.10$ & $1.6 \pm 0.6$ & $<0.7$ & $<1$ & $1.34 \pm 0.29$ \\
\hline $1000-10,000 \ldots$. & $0.23 \pm 0.03$ & $0.37 \pm 0.06$ & $1.4 \pm 0.5$ & $<2$ & $<2$ & $0.82 \pm 0.17$ \\
\hline
\end{tabular}

${ }^{a} F_{1}$ and $F_{2}$ are fluxes for the two sources 3EG J0459+0544 and 3EG J0530+1323, respectively.

${ }^{b}$ Calculated including the propagation of the uncertainties in $A$ and $B$, i.e., $X=B / 2 A\left[1+\left(\sigma_{A} / A\right)^{2}\right]$ and $\sigma_{X}=\sigma_{B} / 2 A\left[1+\left(B \sigma_{A} / A \sigma_{B}\right)^{2}\right]^{1 / 2}$.

${ }^{c}$ The value of the $X$-ratio is very uncertain owing to the uncertainties in $A$ and $B$ 
TABLE 3 OTHER DETERMINATIONS OF THE EMISSIVITY AND $X$-RATIO IN ORION

\begin{tabular}{|c|c|c|c|c|}
\hline Study & $\begin{array}{c}A(>100) \\
\left(10^{-26} \mathrm{~s}^{-1} \mathrm{sr}^{-1}\right)\end{array}$ & $\begin{array}{c}A(>300) \\
\left(10^{-26} \mathrm{~s}^{-1} \mathrm{sr}^{-1}\right)\end{array}$ & $\begin{array}{c}X(>100)^{a} \\
\left(10^{20} \mathrm{~cm}^{-2}\right. \\
\left.\left[\mathrm{K} \mathrm{km} \mathrm{s}^{-1}\right]^{-1}\right)\end{array}$ & $\begin{array}{c}X(>300)^{a} \\
\left(10^{20} \mathrm{~cm}^{-2}\right. \\
\left.\left[\mathrm{K} \mathrm{km} \mathrm{s}^{-1}\right]^{-1}\right)\end{array}$ \\
\hline Bloemen et al. $1984 \ldots \ldots \ldots \ldots . . .$. & $1.70 \pm 0.25^{b}$ & $0.53 \pm 0.30$ & $2.2 \pm 0.6$ & $2.3 \pm 1.6$ \\
\hline Houston \& Wolfendale 1985 & $2.0^{b}$ & & $1.5 \pm 0.4$ & $\ldots{ }^{c}$ \\
\hline DHM $1997 \ldots \ldots \ldots \ldots \ldots \ldots \ldots \ldots$ & $1.68 \pm 0.13$ & $0.73 \pm 0.09$ & $1.06 \pm 0.14$ & $0.9 \pm 0.2$ \\
\hline 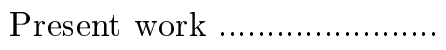 & $1.65 \pm 0.11$ & $0.71 \pm 0.10$ & $1.35 \pm 0.15$ & $1.18 \pm 0.27$ \\
\hline
\end{tabular}

${ }^{a}$ In calculating $X$-ratios, all CO intensities have been corrected to absolute radiation temperatures.

${ }^{b}$ Not determined for the Orion region. Estimated in reference for larger area or for other work.

${ }^{c}$ Not given. 